\title{
Successful treatment of a patient with non-small cell lung cancer and interstitial lung disease with durvalumab: a case report
}

\author{
Haitao Tao ${ }^{1}$, Fangfang $\mathrm{Li}^{2}$, Ruixin $\mathrm{Li}^{1}$, Xiao $\mathrm{Han}^{1}$, Yi Hu${ }^{1}$ \\ ${ }^{1}$ Department of Oncology, The First Medical Center of Chinese PLA General Hospital, Beijing, China; ${ }^{2}$ Department of State Guest, Institute of \\ Health Management, The Second Medical Center of Chinese PLA General Hospital, Beijing, China \\ Correspondence to: Yi Hu. Department of Oncology, The First Medical Center of Chinese PLA General Hospital, Beijing, China. \\ Email: huyi0401@aliyun.com.
}

\begin{abstract}
For patients with advanced non-small cell lung cancer (NSCLC), immune checkpoint inhibitors (ICIs) can offer an effective treatment. However, despite their potential benefits, the use of ICIs can lead to inflammation in various organs, including pneumonitis. Interstitial lung disease (ILD), which is a common complication in patients with NSCLC, can increase the risk of pneumonitis. For NSCLC patients with ILD, the safety of ICIs has yet to be established. Durvalumab is a selective, high-affinity, engineered, human IgG1 monoclonal antibody, which showed durable response and manageable side effects in stage III NSCLC patients after definitive chemoradiotherapy. Pneumonitis in patients who received durvalumab was mostly low grade, given the potential applications in NSCLC with ILD. A 77-year-old man was diagnosed with Stage IV lung squamous carcinoma and ILD at our hospital. The PD-L1 expression assessed by VENTANA PD-L1 (SP263) Assay showed about 60\% of tumor cells exhibit positive. Because the patient refused chemotherapy, he was given durvalumab at $20 \mathrm{mg} / \mathrm{kg}$ every 4 weeks as first-line anti-tumor therapy. After four cycles of therapy, the patient achieved partial remission, and complete remission (CR) had been achieved after 6 cycles of therapy and maintained over two years. No immune-related adverse events were reported. In this case, PD-L1 inhibitors were used to safely treat an NSCLC patient with ILD, which presents the need for further evaluation of their use.
\end{abstract}

Keywords: Non-small cell lung cancer (NSCLC); interstitial lung disease (ILD); immune checkpoint inhibitors (ICIs); pneumonitis; case report

Submitted Apr 17, 2020. Accepted for publication Jun 30, 2020.

doi: 10.21037/apm-20-1134

View this article at: http://dx.doi.org/10.21037/apm-20-1134

\section{Introduction}

Immune checkpoint inhibitors (ICIs) have changed the landscape of treatment for a number of cancers, including non-small cell lung cancer (NSCLC) (1). Several PD-1/ PD-L1 inhibitors including nivolumab, Pembrolizumab and atezolizumab has exhibited better survival with a less toxic profile than docetaxel in second line therapy in NSCLC patients. PD-1/PD-L1 inhibitors combined with chemotherapy or other ICIs has also been approved as first-line treatment for NSCLC patients without oncogenic drivers (2). Although ICIs can effectively achieve the desired antitumor effect by activating the immune system, they also carry the risk of immune-related adverse events (irAEs), including inflammation in various organs $(3,4)$. Pneumonitis, a rare but potentially life-threatening condition, is defined as focal or diffuse inflammation of the lung parenchyma. Previous studies have shown that the incidence of ICIs related pneumonitis ranges from $1 \%$ to $12 \%$ in advanced NSCLC patients (5). The mechanism behind irAEs is still unclear, but prior lung disease is a possible a risk factor of pneumonitis induced by ICIs (6).

Interstitial lung disease (ILD), which is characterized by damage to the lung parenchyma caused by inflammation and fibrosis, is common among smokers (7). Approximately $10 \%$ of NSCLC patients are diagnosed with $\operatorname{ILD}(8,9)$. 


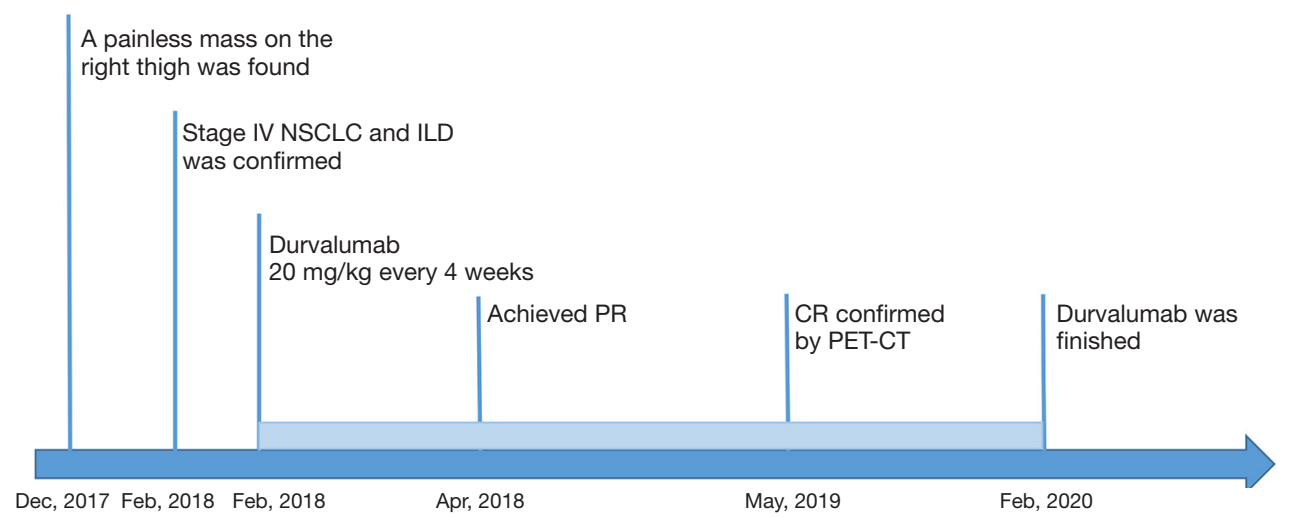

Figure 1 Timeline. The information of the patient with NSCLC and ILD. NSCLC, non-small cell lung cancer; ILD, interstitial lung disease.

Because of the increased risk of pneumonitis, patients with ILD have been excluded from clinical trials involving ICIs. The safety and efficacy of ICIs for treating patients with ILD are not clear.

Durvalumab is a selective, high-affinity, engineered, human IgG1 monoclonal antibody that blocks PD-L1 binding to PD-1 and CD80, which has approved to treat stage III NSCLC, extensive-stage SCLC and Urothelial carcinoma. In PACIFIC study, durvalumab showed durable efficacy and well tolerance in patients with stage III, unresectable NSCLC who did not have disease progression after concurrent chemoradiotherapy. Pneumonitis in patients who received durvalumab was mostly low grade, and the incidence of clinically important grade 3 or 4 events was lower than that in other studies in the same disease context (10). Taken together, durvalumab maybe safety in NSCLC patients with ILD. Here, we report a patient with lung squamous cell carcinoma and ILD who received durvalumab. The patient achieved a good response and experienced no irAEs during more than two years of treatment. We present the following case in accordance with the CARE reporting checklist (available at http:// dx.doi.org/10.21037/apm-20-1134).

\section{Case presentation}

A 77-year-old man was sent to our hospital with a painless mass on the right thigh. The mass measured about $3 \mathrm{~cm} \times 4 \mathrm{~cm}$, and there was no redness or ulceration (Figure 1). Ultrasound-guided biopsy of the mass showed squamous cell carcinoma infiltration in the fibrous tissue,
Immunohistochemistry (IHC) showed CK (+), p63 (+), Ki67 (+40\%), CK5 (+), p40 (+), and Vimentin (-). Metastatic squamous carcinoma was considered. The patient then underwent fluorine-18-fluorodeoxyglucose positron emission tomography/computed tomography $\left({ }^{18} \mathrm{~F}-\mathrm{FDG}\right.$ $\mathrm{PET} / \mathrm{CT}$ ) examination, which showed cancer in the right lower lung, with intramuscular metastases at multiple sites and lymph node metastasis in the mediastinum and mesenteric space (Figure 2). The patient was consequently diagnosed as stage IV lung squamous carcinoma. Chest CT showed reticular opacity and traction bronchiectasis of the bilateral lung, and the diagnosis of ILD was confirmed (Figure 3). The patient had a 30-year history of smoking and no family history of cancer.

PD-L1 expression of tumor cells was evaluated as $60 \%$ positive using the VENTANA PD-L1 (SP263) Assay (Figure 4). As the patient refused chemotherapy, durvalumab monotherapy at $20 \mathrm{mg} / \mathrm{kg}$ every 4 weeks was initiated on January 2018. The patient showed a good response and reached partial remission after four cycles of durvalumab monotherapy (Figure 3). Complete remission was achieved after 6 cycles of therapy and confirmed by another ${ }^{18}$ F-FDG-PET/CT eighteen months later (Figure 2). The patient received durvalumab for two years until January, 2020 and reported no Immune-Related adverse event. Chest CT Examination showed sustained complete remission on March, 2020.

All procedures performed in this case involving human participants were in accordance with the Declaration of Helsinki (as revised in 2013). Informed consent was obtained from the patient. 

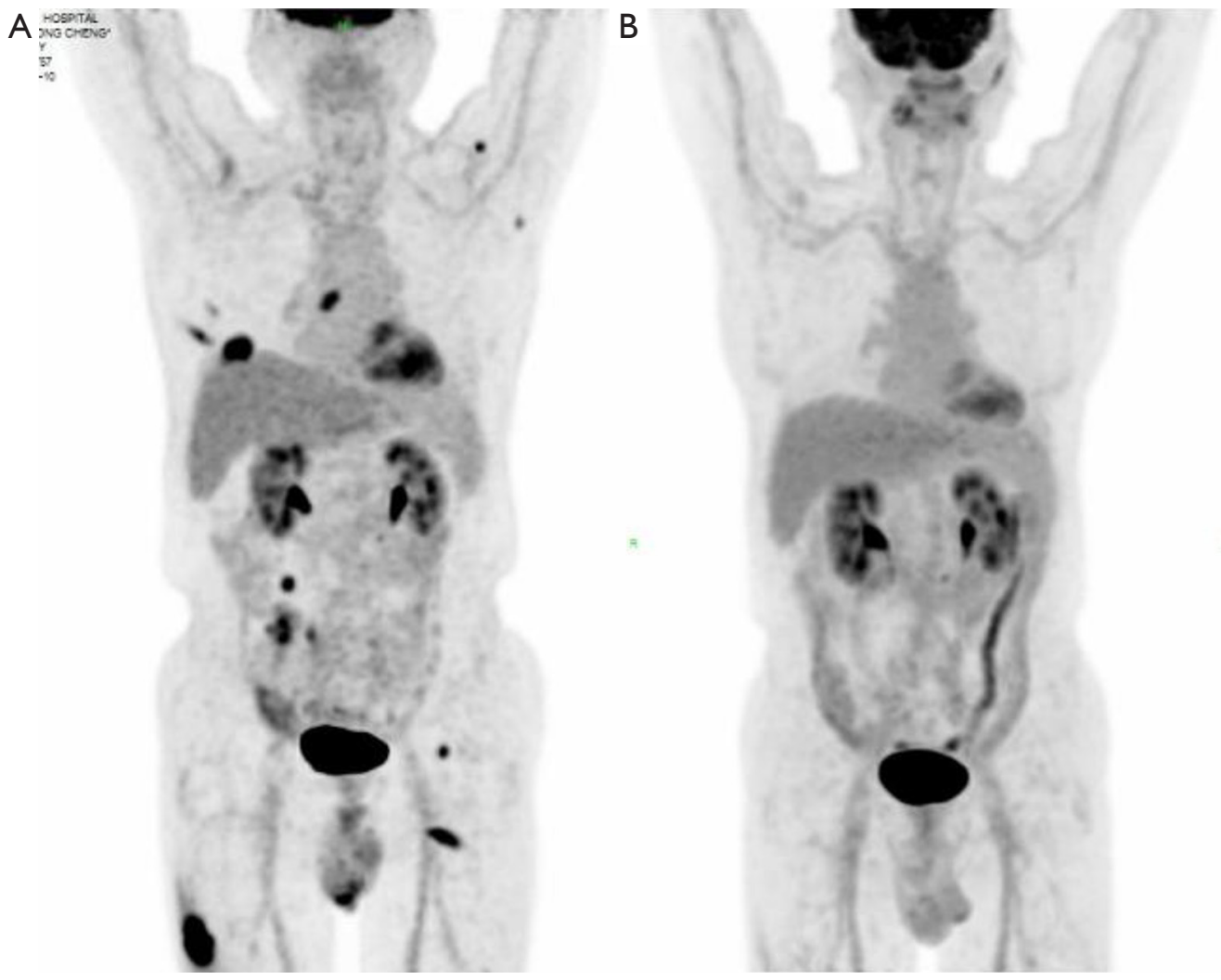

Figure 2 Fluorine-18-fluorodeoxyglucose positron emission tomography/computed tomography $\left({ }^{18}\right.$ F-FDG-PET/CT) of multiple tumors. (A) PET/CT revealed cancer in the right lower lung, with intramuscular metastases at multiple sites and lymph node metastasis in the mediastinum and mesenteric space; (B) 18 months later, PET/CT showed no evidence of the tumor.

\section{Discussion}

We presented the unusual case of an older male patient with advanced NSCLC and ILD who received durvalumab monotherapy as first-line treatment. To the best of our knowledge, NSCLC patients with secondary ILD diagnosis treated with ICIs have rarely been reported.

Monoclonal antibodies against PD-1 and PD-L1 have emerged as effective therapies for patients with advanced NSCLC. The phase 3 MYSTIC study did not meet its primary end points of improved OS with durvalumab $v s$. chemotherapy in patients with $\geq 25 \%$ of tumor cells expressing PD-L1 (11). However, the MYSTIC study's exploratory analysis of the effect of post-study immunotherapy on OS concluded that a high proportion of patients in the chemotherapy arm received subsequent immunotherapy, which may have confounded the primary OS outcome. After adjusting for the effect of subsequent immunotherapy, first-line durvalumab had higher OS benefit compared to chemotherapy $(12,13)$. The rate of grade $\geq 3$ treatment-related AEs was much lower with durvalumab than with chemotherapy. Considering the tolerance, durvalumab was also superior as a first-line therapy for NSCLC patients with tumor cell PD-L1 expression $\geq 25 \%$. The patient in this case report received durvalumab monotherapy as a first-line therapy for NSCLC and achieved sustained CR for more than 2 years, which suggests that durvalumab monotherapy is effective as a firstline treatment for NSCLC.

$\mathrm{PD}-1$ is an inhibitory co-receptor that is expressed on the surface of activated T lymphocytes. PD-1 binds to PDL1 or PD-L2 to modulate T lymphocyte function and prevent autoimmunity in peripheral tissues. Monoclonal antibodies inhibit PD-1/PD-L1 signaling, which not only activates $\mathrm{T}$ lymphocyte-mediated antitumor immunity, but can also result in autoimmune toxicities, some of which can be severe or even fatal. In NSCLC patients, autoimmune pneumonitis, which has been associated with a number of treatment-related deaths in early phase studies, has drawn 

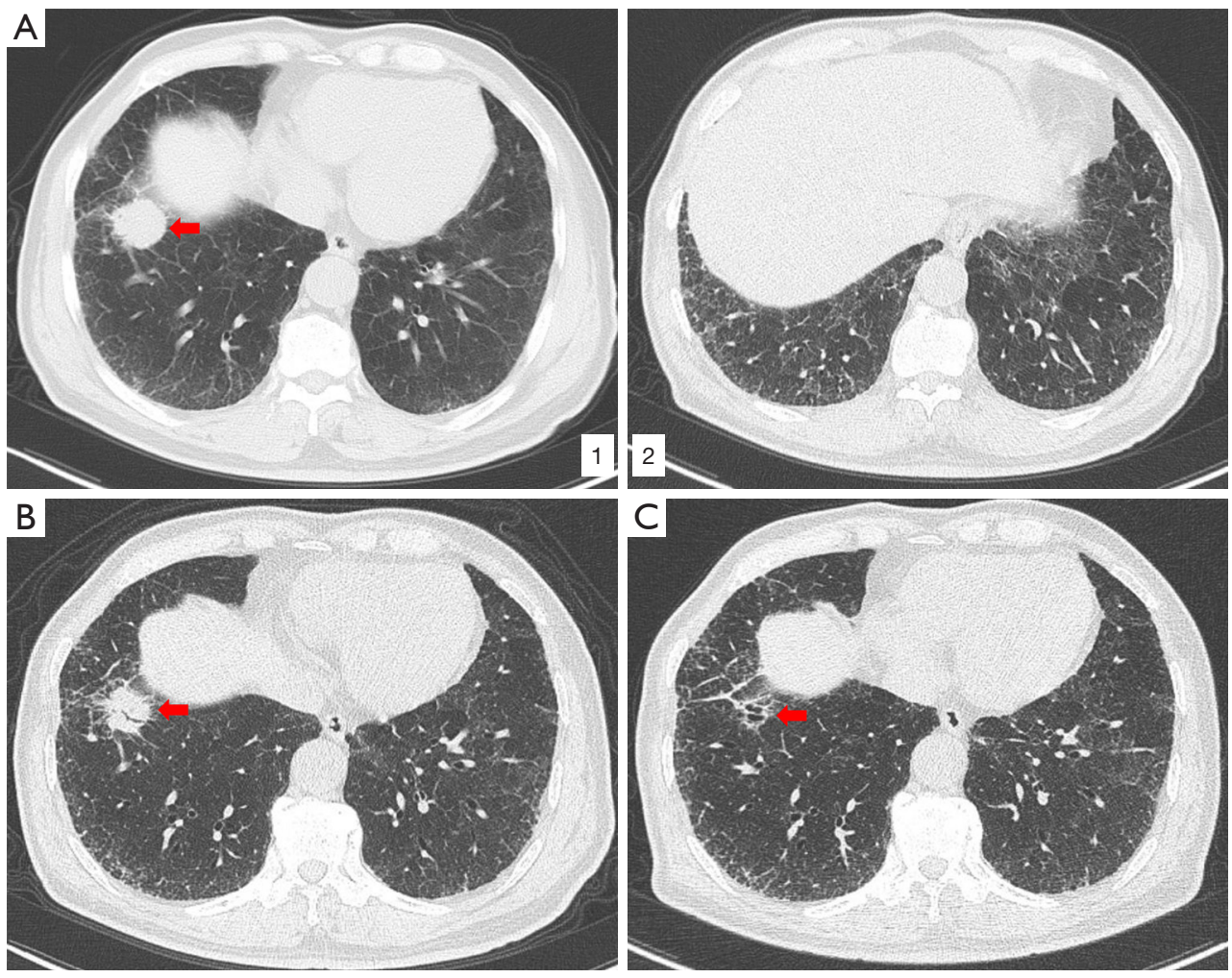

Figure 3 Computed tomography of the primary lung lesion and interstitial lung disease. (A1) The primary lesion in the lung at diagnosis (arrow); (A2) bilateral pulmonary interstitial lung disease; (B) after two cycles of durvalumab, the primary lesion of lung had shrunk (arrow); (C) after four cycles of durvalumab, the primary lesion in the lung had disappeared (arrow).

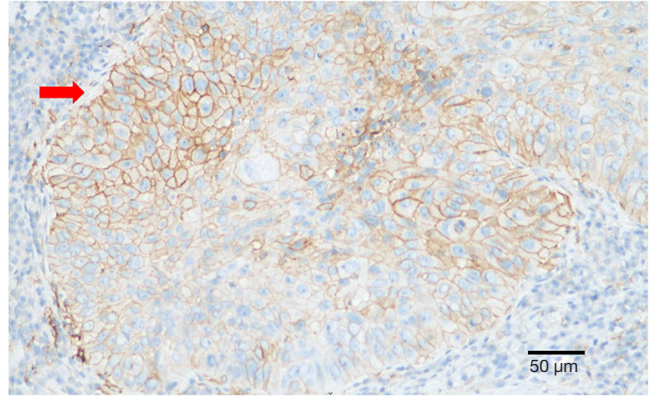

Figure 4 PD-L1 expression. PD-L1 expression was assessed by VENTANA PD-L1 (SP263) Assay. Formalin-fixed, paraffinembedded (FFPE) carcinoma tissue from the tumor on the right thigh was stained with OptiView DAB IHC Detection Kit on a VENTANA BenchMark ULTRA instrument, about $60 \%$ of tumor cells exhibit membrane staining above background (red arrow, $\times 200)$.

particular interest among oncologists $(3,14,15)$. Antibodies against PD-L1 still permit the interaction of PD-1 with its other ligand, PD-L2, which may lessen the blockade effect on the negative inhibitory signals, resulting in reduced autoimmunity. In previous studies, the incidence of highgrade and all-grade pneumonitis was higher with anti-PD-1 therapy but not with anti-PD-L1 therapy when compared to traditional chemotherapy for NSCLC (16). Systematic analysis has also shown that the incidence of grade $3 / 4$ immune-mediated pneumonitis was slightly higher with PD-1 inhibitors than with PD-L1 inhibitors (17).

Approximately $10 \%$ of NSCLC patients are diagnosed with ILD. Since acute exacerbation of ILD is a fatal adverse event that can occur during therapy, treating NSCLC patients with ILD presents a challenge for clinicians. There have been few prospective clinical trials of patients with both NSCLC and ILD. However, due to the low risk of immune-mediated pneumonitis with PD-L1 inhibitors, this patient was successfully treated with durvalumab monotherapy, which indicates that PD-L1 inhibitors can be used to safely treat patients with NSCLC and ILD. However, further investigation into their use is needed. 
In conclusion, we reported a patient with NSCLC and ILD who was successfully treated with durvalumab monotherapy as a first-line therapy and experienced no irAEs. The safety of PD-L1 inhibitors for treating patients with ILD warrants further study.

\section{Acknowledgments}

Funding: None.

\section{Footnote}

Reporting Checklist: The authors have completed the CARE reporting checklist. Available at http://dx.doi.org/10.21037/ apm-20-1134

Conflicts of Interest: All authors have completed the ICMJE uniform disclosure form (available at http://dx.doi. org/10.21037/apm-20-1134). The authors have no conflicts of interest to declare.

Ethical Statement: The authors are accountable for all aspects of the work in ensuring that questions related to the accuracy or integrity of any part of the work are appropriately investigated and resolved. All procedures performed in this case involving human participants were in accordance with the Declaration of Helsinki (as revised in 2013). Informed consent was obtained from the patient.

Open Access Statement: This is an Open Access article distributed in accordance with the Creative Commons Attribution-NonCommercial-NoDerivs 4.0 International License (CC BY-NC-ND 4.0), which permits the noncommercial replication and distribution of the article with the strict proviso that no changes or edits are made and the original work is properly cited (including links to both the formal publication through the relevant DOI and the license). See: https://creativecommons.org/licenses/by-nc-nd/4.0/.

\section{References}

1. Kunimasa K, Nishino K, Kumagai T. Is an immune checkpoint inhibitor really a hopeless therapeutic choice for EGFR-mutant non-small cell lung cancer (NSCLC) patients? Ann Transl Med 2019;7:S32.

2. Yoneda K, Imanishi N, Ichiki Y, Tanaka F. Immune Checkpoint Inhibitors (ICIs) in Non-Small Cell Lung Cancer (NSCLC). J UOEH 2018;40:173-89.
3. Liu Y, Wang H, Deng J, et al. Toxicity of tumor immune checkpoint inhibitors-more attention should be paid. Transl Lung Cancer Res 2019;8:1125-33.

4. Suzuki Y, Karayama M, Uto T, et al. Assessment of Immune-Related Interstitial Lung Disease in NonSmall Cell Lung Cancer Patients Treated with Immune Checkpoint Inhibitors: A Multi-Center Prospective Study. J Thorac Oncol 2020;15:1317-27.

5. Marwitz S. Challenges of predicting immune checkpoint therapy responders in lung cancer. Transl Cancer Res 2019;8:722-4.

6. Cui P, Liu Z, Wang G, et al. Risk factors for pneumonitis in patients treated with anti-programmed death-1 therapy: a case-control study. Cancer Med 2018;7:4115-20.

7. Travis WD, Costabel U, Hansell DM, et al. An official American Thoracic Society/European Respiratory Society statement: Update of the international multidisciplinary classification of the idiopathic interstitial pneumonias. Am J Respir Crit Care Med 2013;188:733-48.

8. Fujimoto D, Morimoto T, Ito J, et al. A pilot trial of nivolumab treatment for advanced non-small cell lung cancer patients with mild idiopathic interstitial pneumonia. Lung Cancer 2017;111:1-5.

9. Fujimoto D, Sato Y, Morimoto T, et al. Programmed Cell Death Ligand 1 expression in non-small-cell lung cancer patients with interstitial lung disease: a matched casecontrol study. Clin Lung Cancer 2018;19:e667-e673.

10. Antonia SJ, Villegas A, Daniel D, et al. Durvalumab after chemoradiotherapy in stage III non-small-cell lung cancer. N Engl J Med 2017;377:1919-29.

11. Rizvi NA, Cho BC, Reinmuth N, et al. Durvalumab with or without tremelimumab vs standard chemotherapy in first-line treatment of metastatic non-small cell lung cancer: the MYSTIC phase 3 randomized clinical trial. JAMA Oncol 2020;6:661-74.

12. Reinmuth N, Cho BC, Lee KH, et al. Effect of post-study immunotherapy (IO) on overall survival (OS) outcome in patients with metastatic (m) NSCLC treated with first-line durvalumab (D) vs chemotherapy (CT) in the phase III MYSTIC study. Ann Oncol 2019;30 Suppl 2:ii77.

13. Cho BC, Reinmuth N, Lee KH, et al. Efficacy and safety of first-line durvalumab (D) +/- tremelimumab (T) vs platinum-based chemotherapy (CT) based on clinical characteristics in patients with metastatic (m) NSCLC: Results from MYSTIC. Ann Oncol 2019;30 Suppl 2:ii79-ii80.

14. Topalian SL, Hodi FS, Brahmer JR, et al. Safety, activity, and immune correlates of anti-PD-1 antibody in cancer. $\mathrm{N}$ 
Engl J Med 2012;366:2443-54.

15. Garon EB, Rizvi NA, Hui R, et al. Pembrolizumab for the treatment of non-small-cell lung cancer. N Engl J Med 2015;372:2018-28.

16. Rahouma M, Baudo M, Yahia $M$, et al. Pneumonitis as a complication of immune system targeting drugs?-a metaanalysis of anti-PD/PD-L1 immunotherapy randomized clinical trials. J Thorac Dis 2019;11:521-34.

17. Pillai RN, Behera M, Owonikoko TK, et al. Comparison of the toxicity profile of $\mathrm{PD}-1$ versus $\mathrm{PD}-\mathrm{L} 1$ inhibitors in non-small cell lung cancer: A systematic analysis of the literature. Cancer 2018;124:271-7.

(English Language Editor: J. Reynolds)

Cite this article as: Tao H, Li F, Li R, Han X, Hu Y. Successful treatment of a patient with non-small cell lung cancer and interstitial lung disease with durvalumab: a case report. Ann Palliat Med 2020;9(5):3623-3628. doi: 10.21037/apm-20-1134 